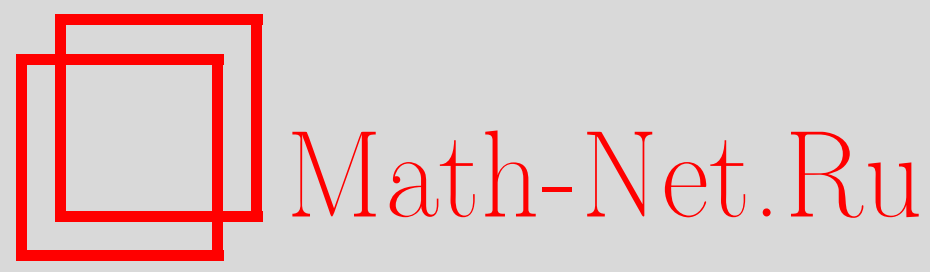

В. Ф. Бабенко, В. А. Кофанов, С. А. Пичугов, Об аддитивных неравенствах для промежуточных производных дифференцируемых отображений банаховых пространств, Матем. заметки, 1998, том 63, выпуск 3, 332-342

DOI: https://doi.org/10.4213/mzm1287

Использование Общероссийского математического портала Math-Net.Ru подразумевает, что вы прочитали и согласны с пользовательским соглашением http://www . mathnet.ru/rus/agreement

Параметры загрузки:

IP : 3.89 .185 .249

26 апреля 2023 г., 04:54:30

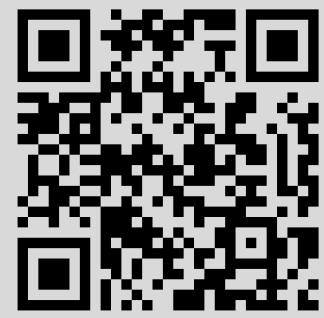




\title{
ОБ АДДИТИВНЫХ НЕРАВЕНСТВАХ ДЛЯ ПРОМЕЖУТОЧНЫХ ПРОИЗВОДНЫХ ДИФФЕРЕНЦИРУЕМЫХ ОТОБРАЖЕНИЙ БАНАХОВЫХ ПРОСТРАНСТВ
}

\author{
В. Ф. Бабенко, В. А. Кофанов, С. А. Пичугов
}

Пусть $X, Y$ - вещественные банаховы пространства, $U \subset X$ - открытое ограниченное множество, звездное относительно некоторой точки, $n, k \in \mathbb{N}, k<n, M_{n, k}(U, Y)$ точная константа в неравенстве типа Маркова для производных полиномиальных отображений. Доказано, что при любом $M \geqslant M_{n, k}(U, Y)$ существует константа $B>0$ такая, что для любой функции $f \in C^{n}(U, Y)$ имеет место неравенство

$$
\|\| f^{(k)}\left\|_{U} \leqslant M\right\| f\left\|_{U}+B\right\| \mid f^{(n)} \|_{U} .
$$

Константа $M=M_{n-1, k}(U, Y)$ является неулучшаемой в том смысле, что $M_{n-1, k}(U$, $Y)=\inf M$, где inf берется по всем $M$ таким, что для некоторого $B>0$ оценка выполняется для всех $f \in C^{n}(U, Y)$.

Библиографой: 15 названий.

1. Пусть $X$ и $Y$ - банаховы пространства над полем $\mathbb{R}$ вешественных чисел. Для любого $n \in \mathbb{N}$ будем через $\mathscr{L}_{n}(X, Y)$ обозначать пространство $n$-линейных (т.е. линейных по каждой переменной при фиксированных остальных переменных) отображений $F: X \rightarrow Y$, где $X^{n}$ - декартово произведение $n$ экземпляров пространства $X$. В частности, $\mathscr{L}(X, Y)=\mathscr{L}_{1}(X, Y)$ - пространство линейных непрерьвных отображений $F: X \rightarrow Y$. Норма отображения $F \in \mathscr{L}_{n}(X, Y)$ определяется равенством

$$
\|F\|_{\mathscr{L}_{n}(X, Y)}:=\sup \left\{\left\|F\left(x_{1}, \ldots, x_{n}\right)\right\|_{Y}: x_{i} \in X,\left\|x_{i}\right\|_{X}=1, i=1, \ldots, n\right\} .
$$

Пусть $U \subset X$ - непустое, открытое, ограниченное множество; $f: U \rightarrow Y$ - отображение класса $C^{n}=C^{n}(U ; Y) ; f^{(k)}-k$-е производное отображение для $f$ (по поводу используемых в данной статье понятий теории дифференцируемых отображений банаховых пространств см. [1, гл. 1]). Как известно, при любом $n \geqslant 2$ имеет место естественная изометрия

$$
\mathscr{L}\left(X ; \mathscr{L}_{n-1}(X, Y)\right) \approx \mathscr{L}_{n}(X, Y)
$$

в силу которой $k$-е производное отображение для $f$ можно трактовать как отображение $f^{(k)}: U \rightarrow \mathscr{L}_{k}(X, Y)$.

Работа выполнена при частичной финансовой поддержке Международного научного фонда, грант U92000, и Государственного комитета Украины по науке и технике.

(C) В.Ф. БАвенко, В. А. КОФАНОВ, С. А. ПичУГОв 1998 
Для $f \in C^{n}(U ; Y)$ положим

$$
\|f f\|_{U}=\sup _{x \in U}\|f(x)\|_{Y}
$$

и $($ при $1 \leqslant k \leqslant n)$

$$
\|\| f^{(k)}\left\|_{U}=\sup _{x \in U}\right\| f^{(k)}(x) \|_{\mathscr{L}_{k}(X, Y)}
$$

В данной статье нас будет интересовать вопрос о существовании постоянных $A$ и $B$ таких, что для любого отображения $f \in C^{n}(U ; Y)$ при фиксированных $n$ и $1 \leqslant k<n$ имеет место неравенство

$$
\left\|\left|f^{(k)}\left\|_{U} \leqslant A\right\|\right| f\left|\left\|_{U}+B\right\|\right| f^{(n)}\right\|_{U},
$$

а также вопрос о точных константах в этом неравенстве. Эти вопросы восходят к работам Ландау [2] и Адамара [3] и для функций $f:(a, b) \rightarrow \mathbb{R}$ исследовались многими авторами (постановки задач, изложение ряда результатов и библиографию можно найти, например, в [4]-[6]). По поводу некоторых результатов, относящихся к числовым функциям многих переменных, см., например, [7].

Мы покажем, что вопрос о принципиальной возможности неравенства (1) тесно связан с вьполнимостью неравенства типа Маркова для полиномиальных отображений $X$ в $Y$ и, более того, точная константа в соответствующем неравенстве типа Маркова дает неулучшаемую константу $A$ в (1).

2. Через $\mathscr{P}_{n}(X, Y)$ будем обозначать пространство полиномиальных отображений (полиномов) степени не вьше $n$, действуюших из $X$ в $Y$, т.е. (см. $[1$, гл. 1]) пространство всех отображений $P_{n}: X \rightarrow Y$ вида

$$
P_{n}(x)=\sum_{j=0}^{n} u_{j}(x, \ldots, x)
$$

где $u_{j} \in \mathscr{L}_{j}(X, Y)$ для $j \geqslant 1$, а $u_{0}$-постоянное отображение $X$ в $Y$ (однородный полином степени 0). Отметим, что если $P_{n} \in \mathscr{P}_{n}(X, Y)$ и $0 \leqslant k \leqslant n$, то $P_{n}^{(k)} \in \mathscr{P}_{n-k}\left(X, \mathscr{L}_{k}(X, Y)\right)$.

Для фиксированных $n, k \in \mathbb{N}, k<n$, и ограниченного открытого множества $U \subset X$ положим

$$
M_{n, k}(U, Y):=\sup \left\{\mid\left\|P_{n}^{(k)}\right\|_{U}: P_{n} \in \mathscr{P}_{n}(X, Y),\left\|P_{n}\right\|_{U} \leqslant 1\right\} .
$$

Если $X=Y=\mathbb{R}$ и $U=(a, b) \subset \mathbb{R}$, то $M_{n, k}(U, Y)$ есть точная константа в неравенстве Маркова [8] для производных алгебраических полиномов. В частности, если

$$
T_{n}(x)=\cos n \arccos x=2^{n-1} x^{n}+\cdots, \quad x \in[-1,1],
$$

- полином Чебышева первого рода, то

$$
M_{n, k}((-1,1), \mathbb{R})=\left\|T_{n}^{(k)}\right\|_{C[-1,1]}=T_{n}^{(k)}(1)=\frac{n^{2}\left(n^{2}-1\right) \cdots\left(n^{2}-(k-1)^{2}\right)}{(2 k-1) ! !}
$$

В общем случае конечность $M_{n, k}(U, Y)$ означает справедливость для полиномов из $\mathscr{P}_{n}(X, Y)$ неравенства типа Маркова

$$
\left\|P_{n}^{(k)}\right\|_{U} \leqslant M\left\|P_{n}\right\|_{U},
$$

и $M_{n, k}(U, Y)$ в этом случае есть точная константа в неравенстве $(2)$.

Очевидно, что если найдутся числа $A$ и $B$ такие, что неравенство (1) имеет место для любого $f \in C^{n}(U, Y)$, то $M_{n-1, k}(U, Y) \leqslant A<\infty$, и следовательно, для полиномов из $\mathscr{P}_{n-1}(X, Y)$ имеет место неравенство $(2)$. Мы покажем, что если множество $U$ является звездным, то верно и обратное утверждение. 
Теорема 1. Пусть $U \subset X$ - открытое, ограниченное множество, звездное относительно некоторой своей точки, $n, k \in \mathbb{N}, k<n$. Если $M_{n-1, k}(U, Y)<\infty$, то найдется $B>0$ такое, что для любого $f \in C^{n}(U, Y)$

$$
\left\|f^{(k)}\right\|_{U} \leqslant M_{n-1, k}(U, Y)\|f\|_{U}+B\left\|f^{(n)}\right\|_{U} .
$$

При этом константа $M_{n-1, k}(U, Y)$ в (3) неулучшаема в том смысле, что

$$
M_{n-1, k}(U, Y)=\inf A,
$$

əде inf берется по всем $A$ таким, что с некоторой константой $B$ неравенство (1) имеет место для всех $f \in C^{n}(U, Y)$.

Отметим, что для функций $f:(a, b) \rightarrow \mathbb{R}$ задача отыскания неулучшаемой в указанном смысле константы $A$ в неравенствах типа (1) изучалась в работах [4], [5], [9]-[11]. В частности, в [11] была отмечена связь такой константы с точной константой в некотором неравенстве типа Маркова-Никольского.

ДоКАЗАТЕЛЬСТВо ТЕОРЕМЫ 1 . Пусть множество $U$ является звездньм относительно точки $a \in U$, и пусть $f \in C^{n}(U, Y)$. Обозначим через $P_{n-1}(f, a)$ связанньй с точкой $a$ полином Тейлора степени $n-1$ для отображения $f$, т.е. для $h \in X$

$$
P_{n-1}(f, a)(h)=f(a)+f^{\prime}(a) \cdot h+\frac{1}{2} f^{\prime \prime}(a) \cdot(h)^{2}+\cdots+\frac{1}{(n-1) !} f^{(n-1)}(a) \cdot(h)^{n},
$$

где для краткости через $(h)^{k}$ обозначен элемент $(h, \ldots, h) \in X^{k}$. Отметим, что для любого $k=1,2, \ldots, n-1$ и любого $h \in X$

$$
P_{n-k-1}\left(f^{(k)}, a\right)(h)=\left(P_{n-1}(f, a)\right)^{(k)}(h) .
$$

Имеем

$$
\begin{aligned}
\left\|f^{(k)}\right\|_{U}= & \sup _{h \in-a+U}\left\|f^{(k)}(a+h)\right\|_{\mathscr{L}_{k}(X, Y)} \\
= & \sup _{h \in-a+U}\left\|f^{(k)}(a+h)-P_{n-k-1}\left(f^{(k)}, a\right)(h)+\left(P_{n-1}(f, a)\right)^{(k)}(h)\right\|_{\mathscr{L}_{k}(X, Y)} \\
\leqslant & \sup _{h \in-a+U}\left\|f^{(k)}(a+h)-P_{n-k-1}\left(f^{(k)}, a\right)(h)\right\|_{\mathscr{L}_{k}(X, Y)} \\
& \quad+\sup _{h \in-a+U}\left\|\left(P_{n-1}(f, a)\right)^{(k)}(h)\right\|_{\mathscr{L}_{k}(X, Y)} .
\end{aligned}
$$

Полагая $f^{(0)}=f$ и применяя формулу Тейлора с остаточным членом в форме Лагранжа (см. [1, теорема 5.6.2]), будем при всех $k=0,1, \ldots, n-1$ и $h \in-a+U$ иметь

$$
\left\|f^{(k)}(a+h)-P_{n-k-1}\left(f^{(k)}, a\right)(h)\right\|_{\mathscr{L}_{k}(X, Y)} \leqslant \frac{\left\|f^{(n)}\right\|\left\|_{U} \cdot\right\| h \|_{X}^{n-k}}{(n-k) !} \leqslant \frac{R_{a}(U)^{n-k}}{(n-k) !}\left\|f^{(n)}\right\|_{U},
$$

где $R_{a}(U)=\sup \left\{\|x-a\|_{X}: x \in U\right\}-$ радиус наименьшего шара с центром в $a$, содержащего множество $U$. 
Далее заметим, что при любом $a \in X M_{n, k}(a+U, Y)=M_{n, k}(U, Y)$. Поэтому

$$
\begin{aligned}
& \sup _{h \in-a+U}\left\|\left(P_{n-1}(f, a)\right)^{(k)}(h)\right\|_{\mathscr{L}_{k}(X, Y)}=\left\|\left(P_{n-1}(f, a)\right)^{(k)}\right\|_{-a+U} \\
& \quad \leqslant M_{n-1, k}(U, Y)\left\|P_{n-1}(f, a)\right\|_{-a+U}=M_{n-1, k}(U, Y) \sup _{h \in-a+U}\left\|P_{n-1}(f, a)(h)\right\|_{Y} \\
& \quad \leqslant M_{n-1, k}(U, Y)\left(\sup _{h \in-a+U}\left\|f(a+h)-P_{n-1}(f, a)(h)\right\|_{Y}+\sup _{h \in-a+U}\|f(a+h)\|_{Y}\right),
\end{aligned}
$$

откуда, используя (4), получаем

$$
\sup _{h \in-a+U}\left\|\left(P_{n-1}(f, a)\right)^{(k)}(h)\right\|_{\mathscr{L}_{k}(X, Y)} \leqslant M_{n-1, k}(U, Y)\left(\frac{R_{a}(U)^{n}}{n !}\left\|f^{(n)}\right\|_{U}+\|f\|_{U}\right) .
$$

Учитывая полученную в начале доказательства оценку для $\left\|f^{(k)}\right\|_{U}$ и неравенства (4), (5), будем иметь

$$
\left\|f^{(k)}\right\|_{U} \leqslant M_{n-1, k}(U, Y)\|f\|_{U}+\left(M_{n-1, k}(U, Y) \frac{R_{a}(U)^{n}}{n !}+\frac{R_{a}(U)^{n-k}}{(n-k) !}\right)\left\|f^{(n)}\right\| \|_{U}
$$

Таким образом, неравенство (3) с константой

$$
B=M_{n-1, k}(U, Y) \frac{R_{a}(U)^{n}}{n !}+\frac{R_{a}(U)^{n-k}}{(n-k) !}
$$

доказано.

Неулучшаемость константы $M_{n-1, k}(U, Y)$ при $\|f\|_{U}$ в (3) легко следует из того, что $P_{n-1}^{(n)}=0$ для любого $P_{n-1} \in \mathscr{P}_{n-1}(X, Y)$.

Теорема доказана.

Из (6) немедленно вытекает

СлЕДСтвиЕ 1. Если U - непустое, открытое, ограниченное, выпуклое множество и

$$
R(U)=\inf _{a \in U} R_{a}(U)
$$

- чебышевский радиус множества $U$, то для константы $B$ в неравенстве (3) справедлива оценка

$$
B \leqslant M_{n-1, k}(U, Y) \frac{R(U)^{n}}{n !}+\frac{R(U)^{n-k}}{(n-k) !} .
$$

Из результатов работы [12] следует, что если $U$ - непустое, открытое, ограниченное, выпуклое множество и

$$
r(U)=\sup \{r \geqslant 0: \exists x \in U, B(x, r) \subset U\},
$$

где $B(x, r)$ - открытый шар радиуса $r$ с центром $x$, то для точной константы $M_{n, 1}(U, Y)$ справедливы оценки

$$
\frac{n^{2}}{r(U)} \leqslant M_{n, 1}(U, Y) \leqslant \frac{4 n^{2}}{r(U)}
$$

где левое неравенство получено при дополнительном предположении, что множество $U$ центрально-симметричное.

Сопоставляя теорему 1 , соотношение (8) и неравенство (7), устанавливаем, что имеет место 
Теорема 2. Если $U \subset X$-непустое, открытое, ограниченное, выпуклое множество, то для любого $f \in C^{n}(U, Y)$ имеет место неравенство

$$
\left\|f^{\prime}\right\|_{U} \leqslant \frac{4(n-1)^{2}}{r(U)}\|f \mid\|_{U}+\left(\frac{4(n-1)^{2}}{r(U)} \frac{R(U)^{n}}{n !}+\frac{R(U)^{n-1}}{(n-1) !}\right)\left\|f^{(n)}\right\| \|_{U} .
$$

В работе [12, теорема 3] доказано неравенство для нормы производной полиномиального отображения $\left\|P_{n}^{\prime}(x)\right\|_{\mathscr{L}_{1}(X, Y)}$, учитьвающего положение точки $x$. А именно, для заданного непустого, открытого, ограниченного, вьпуклого множества $U \subset X$, точки $x_{0} \in U$ и числа $\omega \in(0,1)$ через $U_{\omega}\left(x_{0}\right)$ обозначим $\omega$-с жсатие $U$ относительно точки $x_{0}$, т.e.

$$
U_{\omega}\left(x_{0}\right)=\left\{x \in X: x_{0}+\frac{x-x_{0}}{\omega} \in U\right\} .
$$

Пусть также для $x_{0}, x \in U$

$$
\begin{gathered}
r\left(x_{0}\right)=\sup \left\{r>0: B\left(x_{0}, r\right) \subset U\right\}, \quad \rho\left(x, x_{0}\right)=\inf \left\{\omega \geqslant 0: x \in U_{\omega}\left(x_{0}\right)\right\} \\
b(x)=\inf _{x_{0} \in U} \frac{1}{r\left(x_{0}\right) \sqrt{1-\rho\left(x, x_{0}\right)}} .
\end{gathered}
$$

Тогда [12]

$$
\left\|P_{n}^{\prime}(x)\right\|_{\mathscr{L}_{1}(X, Y)} \leqslant 3 b(x) n\left\|P_{n}\right\|_{U}
$$

Теперь из теоремы 1 и неравенства (9) мы получаем оценку нормы производной отображения $f(x)$ с учетом положения аргумента $x$.

ТЕОрема 3. Пусть $U \subset X$ - непустое, открытое, ограниченное, выпуклое множество. Тогда для данного $n \in \mathbb{N}$ найдется $B>0$ такое, что для любого $f \in C^{n}(U, Y)$ и любого $x \in U$ справедливо неравенство

$$
\left\|f^{\prime}(x)\right\|_{\mathscr{L}_{1}(X, Y)} \leqslant \min \left\{\frac{4(n-1)^{2}}{r(U)}, 3 b(x)(n-1)\right\}\|f \mid\|_{U}+B\|\| f^{(n)}\|\|_{U} .
$$

3. Через $W_{\infty}^{n}\left((-1,1)^{m}\right), m, n \in \mathbb{N}$, обозначим класс вещественнозначных функций $f \in L_{\infty}(-1,1)^{m}$, имеющих для любого мультииндекса $\alpha=\left(\alpha_{1}, \ldots, \alpha_{m}\right) \in \mathbb{Z}_{+}^{m}$ такого, что $|\alpha|=\alpha_{1}+\cdots+\alpha_{m}=n$, обобщенную в смысле Соболева производную $D^{\alpha} f$, принадлежащую $L_{\infty}(-1,1)^{m}$.

Положим для $k \in \mathbb{N}, k \leqslant n$,

$$
\nabla^{k} f=\left(D^{\alpha} f\right)_{|\alpha|=k}, \quad\left\|\nabla^{k} f\right\|_{\infty}=\max _{|\alpha|=k}\left\|D^{\alpha} f\right\|_{L_{\infty}(-1,1)^{m}}
$$

Пусть для $n, k \in \mathbb{N}, k<n$,

$$
M_{n, k}^{*}:=\sup \left\{\left\|\nabla^{k} P_{n}\right\|_{\infty}: P_{n} \in \mathscr{P}_{n, m},\left\|P_{n}\right\|_{L_{\infty}(-1,1)^{m}} \leqslant 1\right\}
$$

где $\mathscr{P}_{n, m}=\mathscr{P}_{n}\left(\mathbb{R}^{m}, \mathbb{R}\right)$ - множество алгебраических полиномов от $m$ переменных, степени не выше $n$ в том смысле, что сумма степеней в каждом мономе не превосходит $n$. 
ТЕОРема 4. Пусть $k, m, n \in \mathbb{N}, k<n$. Тогда для любой функиии $f \in W_{\infty}^{n}\left((-1,1)^{m}\right)$ имеет место неравенство

$$
\left\|\nabla^{k} f\right\|_{\infty} \leqslant M_{n-1, k}^{*}\|f\|_{L_{\infty}(-1,1)^{m}}+B\left\|\nabla^{n} f\right\|_{\infty}
$$

с некоторой константой $B$, не зависящей от $f$. При этом константа $M_{n-1, k}^{*}$ в (10) неулучшаема.

ДокАзАТЕЛьство. Сначала неравенство (10) мы докажем для функций $f$, имеющих в $(-1,1)^{m}$ непрерывные частные производные до порядка $n$ включительно. Такие функции $f$ являются функциями класса $C^{n}\left((-1,1)^{m}, \mathbb{R}\right)$. Тогда в силу теоремы 1 будем иметь

$$
\left\|\left|f^{(k)}\right|\right\|_{(-1,1)^{m}} \leqslant M_{n-1, k}\left((-1,1)^{m}, \mathbb{R}\right)\|\| f\left\|_{(-1,1)^{m}}+B\right\|\left\|f^{(n)} \mid\right\|_{(-1,1)^{m}} .
$$

Отметим (см., например, [13, с. 210], что для $f \in C^{n}\left((-1,1)^{m}, \mathbb{R}\right)$ при любом $0<k \leqslant n$ для любого набора векторов $h^{i}=\left(h_{1}^{i}, \ldots, h_{m}^{i}\right), i=1, \ldots, k$, имеет место равенство

$$
f^{(k)}(x)\left(h^{1}, \ldots, h^{k}\right)=\sum_{\left(j_{1}, \ldots, j_{k}\right)} \frac{\partial^{k}}{\partial x_{j_{1}} \cdots \partial x_{j_{k}}} f(x) h_{j_{1}}^{1} h_{j_{2}}^{2} \cdots h_{j_{k}}^{k}
$$

где сумма распространяется на все $m^{k}$ наборов различных последовательностей $\left(j_{1}\right.$, $\ldots, j_{k}$ ) целых чисел, принадлежащих $[1, m]$.

Считая, что пространство $\mathbb{R}^{m}$ снабжено $l_{1}$-нормой

$$
\|x\|_{1}:=\sum_{j=1}^{m}\left|x_{j}\right|
$$

и вычисляя норму $k$-линейного функционала

$$
f^{(k)}(x): \underbrace{\mathbb{R}^{m} \times \cdots \times \mathbb{R}^{m}}_{k \text { раз }} \rightarrow \mathbb{R},
$$

определяемого соотношением (12), получаем

$$
\begin{aligned}
\left\|f^{(k)}(x)\right\|_{\mathscr{L}_{k}\left(\mathbb{R}^{m}, \mathbb{R}\right)} & =\sup _{\substack{\left\|h^{i}\right\|_{1} \leqslant 1 \\
i=1, \ldots, k}}\left|\sum_{\substack{\left.j_{1}, \ldots, j_{k}\right)\\
}} \frac{\partial^{k}}{\partial x_{j_{1}} \cdots \partial x_{j_{k}}} f(x) h_{j_{1}}^{1} \cdots h_{j_{k}}^{k}\right| \\
& =\max _{\substack{\left(j_{1}, \ldots, j_{k}\right) \\
\text { max }}}\left|\frac{\partial^{k}}{\partial x_{j_{1}} \cdots \partial x_{j_{k}}} f(x)\right| .
\end{aligned}
$$

Следовательно,

$$
\begin{aligned}
\left\|f^{(k)}\right\| \|_{(-1,1)^{m}} & =\sup _{x \in(-1,1)^{m}}\left\|f^{(k)}(x)\right\|_{\mathscr{L}_{k}\left(\mathbb{R}^{m}, \mathbb{R}\right)} \\
& =\left\|\nabla^{k} f\right\|_{\infty}, \quad M_{n-1, k}\left((-1,1)^{m}, \mathbb{R}\right)=M_{n-1, k}^{*} .
\end{aligned}
$$

Теперь из (11) и (13) следует $(10)$ для любой $f \in C^{n}\left((-1,1)^{m}, \mathbb{R}\right)$. 
Пусть теперь $f$ - произвольная функция из $W_{\infty}^{n}\left((-1,1)^{m}\right)$. Продолжим ее на все $\mathbb{R}^{m}$, полагая равной 0 вне $(-1,1)^{m}$, и рассмотрим ее усреднение $f_{\varepsilon}$ по Соболеву $[14$, c. 71$]$

$$
f_{\varepsilon}(x)=\frac{1}{\varepsilon^{m}} \int_{\mathbb{R}^{m}} \varphi\left(\frac{|x-u|}{\varepsilon}\right) f(u) d u, \quad \varepsilon>0
$$

где $\varphi(t):[-1,1] \rightarrow \mathbb{R}_{+}-$бесконечно дифференцируемая, четная, неотрицательная функция, причем

$$
\int_{\mathbb{R}} \varphi(t) d t=1
$$

Тогда $f_{\varepsilon}$ также бесконечно дифференцируема,

$$
\left\|f_{\varepsilon}\right\|_{L_{\infty}(-1,1)^{m}} \leqslant\|f\|_{L_{\infty}(-1,1)^{m}}
$$

и $\left(D^{\alpha} f_{\varepsilon}\right)(x)=\left(D^{\alpha} f\right)_{\varepsilon}(x)$ для всех $\alpha(|\alpha| \leqslant n), x \in(-1+\varepsilon, 1-\varepsilon)^{m}$.

Заметим, что если в $(10)$ область аргументов $(-1,1)^{m}$ заменить на $(-1+\varepsilon, 1-\varepsilon)^{m}$ $(\varepsilon \in(0,1))$, то неравенство $(10)$ для функций $g$ из $W_{\infty}^{n}\left((-1+\varepsilon, 1-\varepsilon)^{m}\right)$ приобретет вид

$$
\left\|\nabla^{k} g\right\|_{\infty, \varepsilon} \leqslant \frac{1}{(1-\varepsilon)^{k}} M_{n-1, k}^{*}\|g\|_{L_{\infty}(-1+\varepsilon, 1-\varepsilon)^{m}}+(1-\varepsilon)^{n-k} B\left\|\nabla^{n} g\right\|_{\infty, \varepsilon}
$$

где

$$
\left\|\nabla^{k} g\right\|_{\infty, \varepsilon}:=\max _{|\alpha|=k}\left\|D^{\alpha} g\right\|_{L_{\infty}(-1+\varepsilon, 1-\varepsilon)^{m}}
$$

(для доказательства этого факта достаточно применить $(10)$ к функции $g\left(y(1-\varepsilon)^{-1}\right.$ ) из $\left.W_{\infty}^{n}\left((-1,1)^{m}\right)\right)$.

Следовательно, для любого $\alpha$ такого, что $|\alpha|=k, k<n$,

$$
\begin{aligned}
& \left\|\left(D^{\alpha} f\right)_{\varepsilon}\right\|_{L_{\infty}(-1+\varepsilon, 1-\varepsilon)^{m}}=\left\|D^{\alpha}\left(f_{\varepsilon}\right)\right\|_{L_{\infty}(-1+\varepsilon, 1-\varepsilon)^{m}} \\
& \quad \leqslant \frac{1}{(1-\varepsilon)^{k}} M_{n-1, k}^{*}\left\|f_{\varepsilon}\right\|_{L_{\infty}(-1+\varepsilon, 1-\varepsilon)^{m}}+(1-\varepsilon)^{n-k} B\left\|\nabla^{n}\left(f_{\varepsilon}\right)\right\|_{\infty, \varepsilon} \\
& \quad \leqslant \frac{1}{(1-\varepsilon)^{k}} M_{n-1, k}^{*}\|f\|_{L_{\infty}(-1+\varepsilon, 1-\varepsilon)^{m}}+(1-\varepsilon)^{n-k} B\left\|\left(\nabla^{n} f\right)_{\varepsilon}\right\|_{\infty, \varepsilon} \\
& \quad \leqslant \frac{1}{(1-\varepsilon)^{k}} M_{n-1, k}^{*}\|f\|_{L_{\infty}(-1,1)^{m}}+(1-\varepsilon)^{n-k} B\left\|\nabla^{n} f\right\|_{L_{\infty}(-1,1)^{m}}
\end{aligned}
$$

Теперь нужно перейти к пределу при $\varepsilon \rightarrow 0$ и учесть тот факт, что функции $D^{\alpha} f$ при $|\alpha|<n$ непрерьвны (это следует из теорем вложения [14, с. 145]), и следовательно, при $\varepsilon \rightarrow 0$

$$
\left\|\left(D^{\alpha} f\right)_{\varepsilon}\right\|_{L_{\infty}(-1+\varepsilon, 1-\varepsilon)^{m}} \rightarrow\left\|D^{\alpha} f\right\|_{L_{\infty}(-1,1)^{m}}
$$

Теорема 4 доказана. 
4. В заключение отметим два частных случая, когда константу $M_{n-1, k}^{*}$ в (10) удается вычислить явно.

Пусть $T_{n-1}(t)=\cos (n-1) \arccos t, t \in(-1,1),-$ полином Чебьшева первого рода. Случай $k=1$.

$$
M_{n-1,1}^{*}=\left\|T_{n-1}^{\prime}\right\|_{L_{\infty}(-1,1)}=T_{n-1}^{\prime}(1)=(n-1)^{2} .
$$

Действительно,

$$
\left\|\nabla P_{n-1}\right\|_{\infty}=\max _{|\alpha|=1}\left\|D^{\alpha} P_{n-1}\right\|_{L_{\infty}(-1,1)^{m}}=\max _{1 \leqslant j \leqslant m}\left\|\frac{\partial}{\partial x_{j}} P_{n-1}\right\|_{L_{\infty}(-1,1)^{m}} .
$$

Применяя к полиному $P_{n-1}(x)$, как к функции от $x_{j}$ (при фиксированных остальных переменных), неравенство Маркова, получаем

$$
\left\|\nabla P_{n-1}\right\|_{\infty} \leqslant(n-1)^{2}\left\|P_{n-1}\right\|_{L_{\infty}(-1,1)^{m}}
$$

так что $M_{n-1,1}^{*} \leqslant(n-1)^{2}$. Полагая $P_{n-1}(x)=T_{n-1}\left(x_{1}\right)$, получаем для $M_{n-1,1}^{*}$ нужную оценку снизу.

Случай $k=n-1$.

$$
M_{n-1, n-1}^{*}=\left\|T_{n-1}^{(n-1)}\right\|_{L_{\infty}(-1,1)}=T_{n-1}^{(n-1)}(1)=2^{n-2}(n-1) ! .
$$

Пусть $x^{\alpha}:=x_{1}^{\alpha_{1}} x_{2}^{\alpha_{2}} \cdots x_{m}^{\alpha_{m}}, \alpha !:=\alpha_{1} ! \alpha_{2} ! \cdots \alpha_{m} !$

$$
P_{n-1}(x)=\sum_{|\alpha| \leqslant n-1} a_{\alpha} x^{\alpha}
$$

Тогда для данного $\alpha,|\alpha|=n-1$,

$$
D^{\alpha} P_{n-1}=D^{\alpha}\left(a_{\alpha} x^{\alpha}\right)=\alpha ! a_{\alpha}
$$

И

$$
\begin{aligned}
& \sup _{\left\|P_{n-1}\right\|_{L_{\infty}(-1,1)^{m}=1}}\left\|D^{\alpha} P_{n-1}\right\|_{L_{\infty}(-1,1)^{m}}=\alpha ! \sup _{\left\|P_{n-1}\right\|_{L_{\infty}(-1,1)^{m}=1}}\left|a_{\alpha}\right| \\
& \quad=\frac{\alpha !}{\inf _{\left|a_{\alpha}\right|=1}\left\|P_{n-1}\right\|_{L_{\infty}(-1,1)^{m}}}=\frac{\alpha !}{\inf _{\left\{a_{\beta}\right\}}\left\|x^{\alpha}-\sum_{|\beta| \leqslant n-1, \beta \neq \alpha} a_{\beta} x^{\beta}\right\|_{L_{\infty}(-1,1)^{m}}} .
\end{aligned}
$$

Следовательно,

$$
M_{n-1, n-1}^{*}=\sup _{|\alpha|=n-1} \frac{\alpha !}{\inf _{\left\{a_{\beta}\right\}}\left\|x^{\alpha}-\sum_{|\beta| \leqslant n-1, \beta \neq \alpha} a_{\beta} x^{\beta}\right\|_{L_{\infty}(-1,1)^{m}}} .
$$

Таким образом, задача вычисления $M_{n-1, n-1}^{*}$ сводится фактически к нахождению при каждом $\alpha,|\alpha|=n-1$, полиномов вида (14) с фиксированньм коэффициентом $a_{\alpha}=1$, наименее уклоняющихся от нуля в равномерной метрике.

Для формулировки соответствующего результата обозначим через $m_{\alpha}$ число ненулевых координат мультииндекса $\alpha \in \mathbb{Z}_{+}^{m}$. 
Лемма. Пусть $\alpha \in \mathbb{Z}_{+}^{m},|\alpha|=n-1$, фиксировано. Тогда

$$
\inf _{\left\{a_{\beta}\right\}}\left\|x^{\alpha}-\sum_{|\beta| \leqslant n-1, \beta \neq \alpha} a_{\beta} x^{\beta}\right\|_{L_{\infty}(-1,1)^{m}}=\left\|T_{\alpha}\right\|_{L_{\infty}(-1,1)^{m}}=\frac{1}{2^{n-1-m_{\alpha}}}
$$

əде

$$
T_{\alpha}(x)=\prod_{j: \alpha_{j} \neq 0} 2^{-\left(\alpha_{j}-1\right)} T_{\alpha_{j}}\left(x_{j}\right)
$$

ДоКАЗАТЕЛЬСТво. Для простоты изложения рассмотрим случай $m=2$. Случай $m>2$ исследуется аналогично. Итак, мы ограничимся доказательством равенств

$$
\begin{aligned}
& \inf _{\left\{a_{\beta_{1} \beta_{2}}\right\}}\left\|x_{1}^{j} x_{2}^{n-1-j}-\sum_{\substack{\beta_{1}+\beta_{2} \leqslant n-1 \\
\left(\beta_{1}, \beta_{2} \neq \neq(j, n-1-j)\right.}} a_{\beta_{1} \beta_{2}} x_{1}^{\beta_{1}} x_{2}^{\beta_{2}}\right\|_{L_{\infty}(-1,1)^{2}} \\
& \quad= \begin{cases}2^{-(n-3)}, & j=1,2, \ldots, n-2, \\
2^{-(n-2)}, & j=0 \text { или } j=n-1 .\end{cases}
\end{aligned}
$$

Пусть сначала $1 \leqslant j \leqslant n-2$. Заметим, что произведение полиномов Чебьшева $2^{-(n-3)} T_{j}\left(x_{1}\right) T_{n-j-1}\left(x_{2}\right)$ имеет вид $(14)$ и при этом $a_{j, n-j-1}=1$. Поэтому левая часть (18) не превосходит величины

$$
2^{-(n-3)}\left\|T_{j}\left(x_{1}\right) T_{n-j-1}\left(x_{2}\right)\right\|_{L_{\infty}(-1,1)^{2}}=2^{-(n-3)} .
$$

Для оценки левой части (18) снизу используем тот факт, что класс аппроксимирующих полиномов в (18) содержится в более широком множестве функций вида

$$
Q\left(x_{1}, x_{2}\right)=\sum_{k=0}^{j-1} x_{1}^{k} \varphi_{k}\left(x_{2}\right)+\sum_{l=0}^{n-2-j} x_{2}^{l} \psi_{l}\left(x_{1}\right)
$$

Поэтому

$$
\begin{aligned}
& \inf _{\left\{a_{\beta_{1} \beta_{2}}\right\}}\left\|x_{1}^{j} x_{2}^{n-1-j}-\sum_{\substack{\beta_{1}+\beta_{2} \leqslant n-1 \\
\left(\beta_{1}, \beta_{2}\right) \neq(j, n-1-j)}} a_{\beta_{1} \beta_{2}} x_{1}^{\beta_{1}} x_{2}^{\beta_{2}}\right\|_{L_{\infty}(-1,1)^{2}} \\
& \quad \inf _{\left\{\varphi_{k}, \psi_{l}\right\} \subset L_{\infty}(-1,1)}\left\|x_{1}^{j} x_{2}^{n-1-j}-\left(\sum_{k=0}^{j-1} x_{1}^{k} \varphi_{k}\left(x_{2}\right)+\sum_{l=0}^{n-2-j} x_{2}^{l} \psi_{l}\left(x_{1}\right)\right)\right\|_{L_{\infty}(-1,1)^{2}}
\end{aligned}
$$

Нетрудно видеть (доказательство аналогичного факта см., например, в [15]), что последняя величина равна

$$
\inf _{\left\{b_{k}, c_{l}\right\}}\left\|\left(x_{1}^{j}-\sum_{k=0}^{j-1} b_{k} x_{1}^{k}\right)\left(x_{2}^{n-1-j}-\sum_{l=0}^{n-2-j} c_{l} x_{2}^{l}\right)\right\|_{L_{\infty}(-1,1)^{2}},
$$


а эта нижняя грань, в свою очередь, равна

$$
\left\|\left(2^{-(j-1)} T_{j}\left(x_{1}\right)\right)\left(2^{-(n-j-2)} T_{n-j-1}\left(x_{2}\right)\right)\right\|_{L_{\infty}(-1,1)^{2}}=2^{-(n-3)},
$$

и первое из равенств (18) доказано.

Пусть теперь $j=0$ (случай $j=n-1$ аналогичен). Тогда

$$
\begin{aligned}
2^{-(n-2)} & =\inf _{\left\{a_{l}\right\}}\left\|x_{2}^{n-1}-\sum_{l=0}^{n-2} a_{l} x_{2}^{l}\right\|_{L_{\infty}(-1,1)} \\
& \geqslant \inf _{\left\{a_{\beta_{1} \beta_{2}}\right\}}\left\|x_{2}^{n-1}-\sum_{\substack{\beta_{1}+\beta_{2} \leqslant n-1 \\
\left(\beta_{1}, \beta_{2}\right) \neq(0, n-1)}} a_{\beta_{1} \beta_{2}} x_{1}^{\beta_{1}} x_{2}^{\beta_{2}}\right\|_{L_{\infty}(-1,1)^{2}} \\
& \geqslant \inf _{\left\{\psi_{l}\right\} \subset L_{\infty}(-1,1)}\left\|x_{2}^{n-1}-\sum_{l=0}^{n-2} x_{2}^{l} \psi_{l}\left(x_{1}\right)\right\|_{L_{\infty}(-1,1)^{2}} \\
& \geqslant \sup _{x_{1} \in(-1,1)} \inf _{\left\{\psi_{l}\right\} \subset L_{\infty}(-1,1)} \sup _{x_{2} \in(-1,1)}\left|x_{2}^{n-1}-\sum_{l=0}^{n-2} x_{2}^{l} \psi_{l}\left(x_{1}\right)\right| \\
& \geqslant \sup _{x_{1} \in(-1,1) x_{2} \in(-1,1)} \sup \left|2^{-(n-2)} T_{n-1}\left(x_{2}\right)\right|=2^{-(n-2)},
\end{aligned}
$$

т.е. на самом деле эта цепочка неравенств превращается в цепочку равенств. Соотношения (18) доказаны.

Из этой леммы и соотношения (15) вьводим

СЛЕДСТВИЕ 2. Если $|\alpha|=n-1$, mo

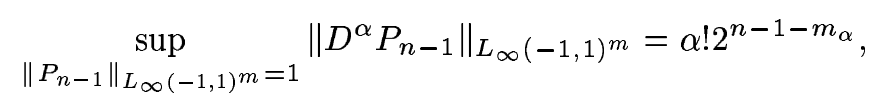

причем верхняя грань достигается на полиноме (17).

Из равенства (16) следует, что

$$
\begin{aligned}
M_{n-1, n-1}^{*} & =2^{n-1} \sup _{|\alpha|=n-1}\left(2^{-m_{\alpha}} \alpha !\right)=2^{n-1} \max _{1 \leqslant k \leqslant \min (m, n-1)} 2^{-k} \max _{\substack{\sum_{j=1}^{m} \alpha_{j}=n-1 \\
m_{\alpha}=k}} \alpha ! \\
& =2^{n-1} \max _{1 \leqslant k \leqslant \min (m, n-1)} 2^{-k} \max _{\sum_{j=1}^{k} \alpha_{j}=n-1} \alpha_{1} ! \alpha_{2} ! \cdots \alpha_{k} ! .
\end{aligned}
$$

Из очевидного неравенства $\alpha_{1} ! \alpha_{2} ! \leqslant\left(\alpha_{1}+\alpha_{2}-1\right)$ ! следует, что

$$
\alpha_{1} ! \alpha_{2} ! \cdots \alpha_{k} ! \leqslant\left(\sum_{j=1}^{k} \alpha_{j}-(k-1)\right) ! .
$$

Поэтому

$$
M_{n-1, n-1}^{*}=2^{n-1} \max _{1 \leqslant k \leqslant \min (m, n-1)} 2^{-k}(n-k) !
$$


Так как функция $\varphi(k)=2^{-k}(n-k)$ ! является убывающей, то

$$
M_{n-1, n-1}^{*} \leqslant 2^{n-1} \varphi(1)=2^{-(n-1)} 2^{-1}(n-1) !=2^{n-2}(n-1) ! .
$$

Легко видеть, что на самом деле выполняется равенство

$$
M_{n-1, n-1}^{*}=2^{n-2}(n-1) !
$$

Действительно, из определения $M_{n-1, n-1}^{*}$ следует, что

$$
M_{n-1, n-1}^{*} \geqslant\left\|D^{(n-1,0, \ldots, 0)} T_{n-1}\left(x_{1}\right)\right\|_{L_{\infty}(-1,1)^{m}}=2^{n-2}(n-1) ! .
$$

Отметим, что задача о точных константах в аддитивных неравенствах для различных $L_{p \text {-норм }} \nabla^{k} f, f, \nabla^{n} f$ рассматривалась в [7].

\section{СПИСОК ЦИТИРОВАННОЙ ЛИТЕРАТУРЫ}

[1] Картан А. Дифференциальное исчисление. Дифференциальные формы. М.: Мир, 1971.

[2] Landau E. Einige Ungleichungen für zweimal differenzierbare Functionen // Proc. London Math. Soc. 1913. V. 13. P. 43-49.

[3] Hadamard J. Sur le module maximum d'une fonction et de ses derivées // C. R. Soc. Math. France. 1914. V. 41. P. 68-72.

[4] Бурёнков В.И. О точных постоянных в неравенствах для норм промежуточных производных на конечном интервале // Тр. МИАН. 1980. Т. 156. С. 22-29.

[5] Бурёнков В.И. О точных постоянных в неравенствах для норм промежуточных производных на конечном интервале. II // Тр. МИАН. 1986. Т. 173. С. 38-49.

[6] Shadrin A. Yu. To the Landau-Kolmogorov problem on a finite interval // Proc. Intern. Conf. (Voneshta Voda, June 18-24), 1993. P. 192-204.

[7] Бурёнков В. И., Гусаков В. А. О точных константах в теоремах вложения Соболева. III // Тр. МИАН. 1993. Т. 204. С. 68-80.

[8] Марков В.А. О функциях, наименее уклоняющихся от нуля в данном промежутке: СПб, 1892.

[9] Шадрин А. Ю. О точных константах в неравенствах между $L_{\infty}$-нормами производных на конечном интервале // Докл. РАН. 1992. Т. 326. С. 53-56.

[10] Бабенко В.Ф., Кофанов В.А., Пичугов С. А. О неравенствах для норм промежуточных производных на конечном интервале // Укр. матем. ж. 1995. Т. 47. № 1. С. 105-107.

[11] Бабенко В. Ф., Кофанов В. А., Пичугов С. А. О неравенствах для норм производных на отрезке // Международная конференция "Теория приближений и задачи вычислительной математики" (Днепропетровск, 26-28 мая). Тезисы докл. Днепропетровск, 1993. С. 12.

[12] Андрианов А.В. Аналоги неравенств А. Маркова и С. Бернштейна для многочленов в банаховых пространствах // Матем. заметки. 1992. Т. 52. № 5. С. 15-20.

[13] Дьедонне ЖК. Основы современного анализа. М.: Мир, 1964.

[14] Бесов О. В., Ильин В. П., Никольский С. М. Интегральные представления функций и теоремы вложения. М.: Наука, 1975.

[15] Пичугов С. А. Точные оценки приближения в $L_{p}$ функциями вида $\varphi(x)+\psi(y) / /$ Укр. матем. ж. 1990. Т. 42. №1. С. 122-125. 